\title{
Vladimír Kohout
}

Sur la déformation projective de certaines surfaces à réseau conjugué dans $S_{2 n+1}$

Czechoslovak Mathematical Journal, Vol. 11 (1961), No. 4, 516-520

Persistent URL: http://dml.cz/dmlcz/100482

\section{Terms of use:}

(C) Institute of Mathematics AS CR, 1961

Institute of Mathematics of the Czech Academy of Sciences provides access to digitized documents strictly for personal use. Each copy of any part of this document must contain these Terms of use.

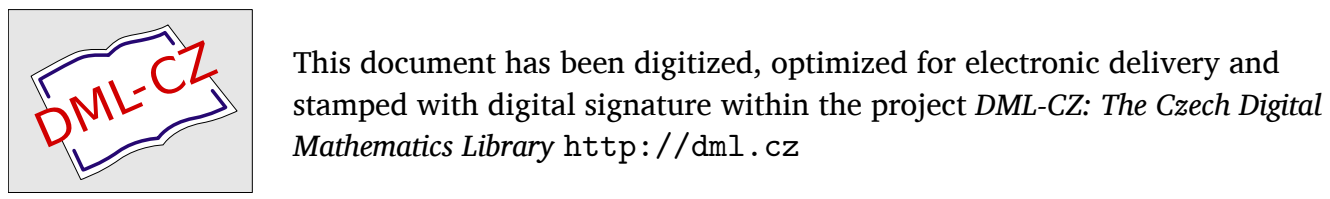




\title{
SUR LA DÉFORMATION PROJECTIVE DE CERTAINES SURFACES A RÉSEAU CONJUGUÉ DANS $S_{2 n+1}$
}

\author{
Vladimír Kohout, Praha
}

(Reçu le 16 mai 1960)

\begin{abstract}
On montre qu'une surface à réseau conjugué dans $S_{2 n+1}$, dont les deux transformées de Laplace dégénèrent, est projectivement déformable d'ordre $n+1$ si et seulement si sa dualisation est du même type.
\end{abstract}

Soit donnée, dans l'espace projectif de dimension impaire $2 n+1$ une surface à réseau conjugué dont les transformées de Laplace dégénèrent en courbes. Supposons ensuite que la surface ait en chacun de ses points un espace osculateur d'ordre $n$ à $2 n$ dimensions et que les espaces osculateurs d'ordre $n+1$ des courbes du réseau conjugué passant par un point se rencontrent en une droite qui n'est pas située dans l'espace osculateur d'ordre $n$ de la surface en ce point. Sous ces hypothèses, il est possible de trouver l'expression analytique de la surface vérifiant le système (voir [1])

$$
\begin{aligned}
x^{1,1} & =0, \\
x^{0, n+1} & =m x+\sum_{i=1}^{n} n_{i} x^{i, 0}+\sum_{i=1}^{n} p_{i} x^{0, i}+q x^{n+1,0}, \quad q \neq 0,
\end{aligned}
$$

les conditions de l'intégrabilité étant

$$
\begin{aligned}
& m^{1,1}+p_{n}^{1,0}-\varepsilon m^{1,0}=0, \\
& m^{01}+n_{1}^{1,1}+p_{n}^{1,0} n_{1}-\varepsilon m-\varepsilon n_{1}^{1,0}=0, \\
& m^{1,0}+p_{1}^{1,1}+p_{n}^{1,0} p_{1}-\varepsilon q_{1}^{1,0}=0, \\
& n_{i}^{1,1}+n_{i-1}^{0,1}+p_{n}^{1,0} u_{i}-\varepsilon n_{i}^{1,0}-\varepsilon n_{i-1}=0 \quad(i=2, \ldots, n), \\
& p_{i}^{1,1}+p_{i-1}^{1,0}+p_{n}^{1,0} p_{i}-\varepsilon p_{i}^{1,0}=0 \quad(i=2, \ldots, n), \\
& n_{n}^{0,1}+p_{n}^{1,0} q+q^{1,1}-\varepsilon n_{n}-\varepsilon q^{1,0}=0, \quad \varepsilon=\frac{q^{0,1}}{q} .
\end{aligned}
$$

Dans ce qui suit, nous entendons par „,surface“ toujours une surface de ce type.

Nous appellerons dualisation de notre surface la surface de l'espace duel décrite par les espaces osculateurs d'ordre $n$ de la surface (ce sont des hyperplans). 
Posons maintenant la question: Quand la dualisation de la surface a-t-elle un réseau conjugué et quand dégénèrent ses transformées de Laplace?

Désignons par le symbole $\left[y_{0}, y_{1}, \ldots, y_{2 n}\right]$ l'hyperplan analytique contenant les points analytiques variables $y_{0}, y_{1}, \ldots, y_{2 n}$; alors $\xi=\left[x, x^{1,0}, x^{0,1}, \ldots, x^{n, 0}, x^{0, n}\right]$ est l'expression analytique de la dualisation de la surface $x$.

On trouve aisément que

$$
\begin{aligned}
\xi^{1,0}= & {\left[x, x^{1,0}, x^{0,1} x^{n+1,0}, \ldots, x^{0, n}\right], } \\
\xi^{0,1}= & {\left[x, x^{1,0}, x^{0,1}, \ldots, x^{n, 0}, x^{0, n+1}\right]=p_{n}\left[x, x^{1,0}, x^{0,1}, \ldots, x^{n, 0}, x^{0, n}\right]+} \\
& +\left[x, x^{1,0}, x^{0,1}, \ldots, x^{n, 0}, x^{n+1,0}\right]= \\
= & p_{n} \xi+q\left[x, x^{1,0}, x^{0,1}, \ldots, x^{n, 0}, x^{n+1,0}\right], \\
\xi^{1,1}= & {\left[x, x^{1,0}, x^{0,1}, \ldots, x^{n+1,0}, x^{0, n+1}\right]=} \\
= & {\left[x, x^{1,0}, x^{0,1}, \ldots, x^{n+1,0}, m x+\sum n_{i} x^{i, 0}+\sum p_{i} x^{0, i}+q x^{n+1,0}\right]=} \\
= & --n_{n}\left[x, x^{1,0}, x^{0,1}, \ldots, x^{n, 0}, x^{n+1,0}\right]+p_{n} \xi^{1,0},
\end{aligned}
$$

d'où

$$
\xi^{1,1}=p_{n} \xi^{1,0}-\frac{n_{n}}{q} \xi^{0,1}+\frac{n_{n} p_{n}}{q} \xi
$$

Donc $\xi$ vérifie l'équation de Laplace, c'est-à-dire sa dualisation a un réseau conjugué.

Passons maintenant au problème de la dégénération des transformées laplaciennes de la dualisation. Pour que les transformées laplaciennes de la dualisation dégénèrent, il faut et il suffit que les invariants $h$ et $k$ de l'équation (3) s'annulent

$$
\begin{aligned}
& h=p_{n}^{1,0}+\frac{p_{n} n_{n}}{q}-\frac{p_{n} n_{n}}{q}=p_{n}^{1,0}=0, \\
& k=\left(-\frac{n_{n}}{q}\right)^{0,1}+\frac{p_{n} n_{n}}{q}-\frac{n_{n} p_{n}}{q}=\left(\frac{n_{n}}{q}\right)^{0,1}=0 .
\end{aligned}
$$

Il s'en ensuit inmédiatement $p_{n}=V_{n}, n_{n}=\widetilde{U} q$. (Les lettres $U, V$, marquées, au besoin, d'indices désignent des fonctions d'une variable $u$, ou $v$ resp. Il résulte de $(26)$ que $q=U V$; écrivons $n_{n}=\bar{U} q=U_{n} V$. Les autres conditions d'intégrabilité se réduisent à

$$
\begin{aligned}
& m^{1,1}-\varepsilon m^{1,0}=0, \\
& m^{0,1}+n_{1}^{1,1}=\varepsilon m+\varepsilon n_{1}^{1,0}, \\
& m^{1,0}+p_{1}^{1,1}=\varepsilon p_{1}^{1,0}, \\
& n_{i}^{1,0}+n_{i-1}^{0,1}-\varepsilon n_{i}^{1,0}-\varepsilon n_{i-1}=0 \quad(i=2, \ldots, n), \\
& p_{i}^{1,1}+p_{i-1}^{1,0}-\varepsilon p_{i}^{1,0}=0 \quad(i=2, \ldots, n), \quad \varepsilon=\frac{V^{\prime}}{V} .
\end{aligned}
$$


Il découle de $\left(4_{4}\right)$ que (pour $i=n$ )

$$
U_{n}^{\prime} V^{\prime}+n_{n-1}^{0,1}=\frac{V^{\prime}}{V} U_{n}^{\prime} V+\frac{V^{\prime}}{V} n_{n-1},
$$

d'où $n_{n-1}=U_{n-1} V$; pour $i=n-2, \ldots, 1$, nous trouvons à partir de (4) successivement

$$
n_{n-2}=U_{n-2} V, \ldots, \quad n_{1}=U_{1} V .
$$

D'une manière analogue, $\left(4_{5}\right)$ nous donne

$$
p_{n-1}=V_{n-1}, \ldots, \quad p_{1}=V_{1} \text {. }
$$

A présent, il résulte de $\left(4_{3}\right)$ que l'on a $m=\tilde{V}$ et de $\left(4_{2}\right)$

$$
\tilde{V}^{\prime}+U_{1}^{\prime} V^{\prime}=\frac{V^{\prime}}{V} \tilde{V}+\frac{V^{\prime}}{V} U_{1}^{\prime} V, \quad \frac{\tilde{V}^{\prime}}{\tilde{V}}=\frac{V^{\prime}}{V},
$$

c'est-à-dire

$$
m=\tilde{V}=\alpha V, \quad \alpha=\text { const. }
$$

Nous voilà arrivés au résultat suivant:

Les transformées laplaciennes de la dualisation de la surface dégénèrent si et seulement si l'on peut trouver pour la surface une expression analytique vérifiant le système

$$
x^{1,1}=0, \quad x^{0, n+1}=\alpha V x+\sum_{i=1}^{n} U_{i} V x^{i, 0}+\sum_{i=1}^{n} V_{i} x^{0, i}+U V x^{n+1,0} .
$$

En changeant les paramètres on peut obtenir $U=V=1$, de sorte que pour l'expression analytique de la surface on aura le système

$$
x^{1,1}=0, \quad x^{0, n+1}=\alpha x+\sum_{i=1}^{n} U_{i} x^{i, 0}+\sum_{i=1}^{n} V_{i} x^{0, i}+x^{n+1,0} .
$$

Les surfaces données par le système (5), ou (6), seront appelées surfaces $R$. Ce sont les surfaces (et elles seules) qui admettent une déformation projective d'ordre $n+1$ (voir [1]).

Cherchons maintenant à établir le système pour la dualisation et envisageons les surfaces $R$ qui sont en déformation projective d'ordre $n+1$ avec leur dualisation.

Posons $\xi=\lambda \bar{\xi}$, où $\lambda$ vérifie les équations

$$
\lambda^{0,1}-V_{n} \lambda=0, \quad \lambda^{1,0}+U_{n} \lambda=0 .
$$

Par cette transformation l'équation (3) devient $\bar{\xi}^{1,1}=0$.

Les relations suivantes peuvent être vérifiées par la méthode de l'induction mathématique

(7) $\bar{\xi}^{n+1,0}=(-1)^{n} \alpha \bar{\xi}+\sum_{\alpha=1}^{n}{ }^{n} A_{\alpha} \bar{\xi}^{\alpha, 0}+\frac{1}{\lambda}\left[x^{1,0}, x^{2,0}, x^{0,1}, x^{3,0}, \ldots, x^{n+1,0}, x^{0, n}\right]$, 
où ${ }^{i} A_{j}$ sont des fonctions de la variable $u$ données par les formules

(8)

$$
\begin{aligned}
& { }^{k} A_{1}={ }^{k-1} A_{1}^{\prime}+(-1)^{k-1} U_{n-k+1}, \\
& { }^{k} A_{\alpha}={ }^{k-1} A_{\alpha}^{\prime}+{ }^{k-1} A_{\alpha-1}, \\
& { }^{k} A_{k}=U_{n}, \quad{ }^{0} A_{1}=0, \quad 2 \leqq \alpha \leqq k-1 ; 1 \leqq k \leqq n .
\end{aligned}
$$

De façon analogue, nous trouvons

$$
\begin{aligned}
& \bar{\xi}^{0, n+1}=(-1)^{n+1} \alpha \bar{\xi}+\sum_{\alpha=1}^{n}{ }^{n} B_{\alpha} \bar{\xi}^{0, \alpha}+\alpha \bar{\xi}+ \\
& +\frac{1}{\lambda}\left[x^{1,0}, x^{2,0}, x^{0,1}, x^{3,0}, \ldots, x^{n+1,0}, x^{0, n}\right],
\end{aligned}
$$

où ${ }^{n} \beta_{\alpha}$ sont des fonctions de $v$ données par les formules

$$
\begin{aligned}
& { }^{k} B_{1}={ }^{k-1} B_{1}^{\prime}+(-1)^{k} V_{n-k+1}, \\
& { }^{k} B_{\alpha}={ }^{k-1} B_{\alpha}^{\prime}+{ }^{k-1} B_{\alpha-1}, \\
& { }^{k} B_{k}=-V_{n}, \quad{ }^{0} B_{1}=0, \quad 2 \leqq \alpha \leqq k-1,1 \leqq k \leqq n .
\end{aligned}
$$

En comparant (7) et (9) nous obienons l'équation de la dualisation (écrite sans barres)

$$
\xi^{0, n+1}=\xi \alpha\left(1-2(-1)^{n}\right)+\sum_{\alpha=1}^{n}{ }^{n} B_{\alpha} \xi^{0, \alpha}-\sum_{\alpha=1}^{n}{ }^{n} A_{\alpha} \xi^{\alpha, 0}+\xi^{n+1,0}
$$

D'après [1], la surface sera en déformation projective non-triviale, d'ordre $n+1$, avec sa dualisation si et seulement si

$$
\alpha \neq 0, \quad-{ }^{n} A_{i}=U_{i}, \quad{ }^{n} B_{i}=V_{i} \quad(i=1, \ldots, n) .
$$

La surface sera en homographie avec sa dualisation si et seulement si

$$
\alpha=0, \quad-{ }^{n} A_{i}=U_{i}, \quad{ }^{n} B_{i}=V_{i} .
$$

\section{Littérature}

[1] A. Švec: Les surfaces $R$ dans les espaces projectifs de dimension impaire. Чех. мат. ж. 9 (84), 1959, 243-264. 


\title{
О ПРОЕКТИВНОМ ИЗГИБАНИИ НЕКОТОРЫХ ПОВЕРХНОСТЕЙ С СОПРЯЖЕННОЙ СЕТЬЮ В $S_{2 n+1}$
}

\author{
ВЛАДИМИР когоуТ (Vladimír Kohout), Прага
}

Поверхности проективного пространства $S_{2 n+1}(n \geqq 1)$, дуализации которых исследуются в этой статье, обладают следующими свойствами: На них существует одна единственная сопряженная сеть, их лапласовские преобразования вырождаются в кривые, а соприкасающиеся пространства порядка $n$ имеют размерность $2 n$. Аналитически эти поверхности выражаются при помоши системы вида (1).

Дуализации таких поверхностей описываются соприкасающимися пространствами порядка $n$ этих поверхностей.

Мы получаем следующие результаты:

1. Дуализачия поверхности указанного типа имеет также сопряженную cemb.

2. Лапласовские преобразования дуализачии вырождаются в кривые, если и только если исходная поверхность допускает изгибание порядка $n+1$.

3. Аналитическое представление дуализачии удовлетворяет системе вида (11).

4. Поверхность связана со своей дуализацией нетривиальным изгибанием порядка $n+1$, если и только если имеет место (12). Поверхность коллинеарна своей дуализачии, если и только если имеет место (13). 